\title{
Some results on a viscosity splitting algorithm in Hilbert spaces
}

Yunpeng Zhang*

\section{"Correspondence:} zhangypliyl@yeah.net

College of Electric Power, North China University of Water Resources and Electric Power, Zhengzhou, 450011, China

\begin{abstract}
In this paper, a viscosity splitting for common solution problems is proposed. Strong convergence theorems are obtained in the framework of Hilbert spaces. Applications are also provided to support the main results.
\end{abstract}

Keywords: zero point; fixed point; variational inclusion; nonexpansive mapping

\section{Introduction; preliminaries}

In this paper, we always assume that $H$ is a real Hilbert space with the inner product $\langle x, y\rangle$ and the induced norm $\|x\|=\sqrt{\langle x, x\rangle}$ for $x, y \in H$. Recall that a set-valued mapping $M: H \rightrightarrows$ $H$ is said to be monotone iff, for all $x, y \in H, f \in M x$, and $g \in M y$ imply $\langle x-y, f-g\rangle \geq 0$. In this paper, we use $M^{-1}(0)$ to denote the zero point set of $M$. A monotone mapping $M: H \rightrightarrows H$ is maximal iff the graph $\operatorname{Graph}(M)$ of $M$ is not properly contained in the graph of any other monotone mapping. It is well known that a monotone mapping $M$ is maximal if and only if, for any $(x, f) \in H \times H,\langle x-y, f-g\rangle \geq 0$, for all $(y, g) \in \operatorname{Graph}(M)$ implies $f \in M x$. For a maximal monotone operator $M$ on $H$, and $r>0$, we may define the singlevalued resolvent $J_{r}: H \rightarrow \operatorname{Dom}(M)$, where $\operatorname{Dom}(M)$ denotes the domain of $M$. It is well known that $J_{r}$ is firmly nonexpansive, and $M^{-1}(0)=F\left(J_{r}\right)$.

The proximal point algorithm, which was proposed by Martinet $[1,2]$ and generalized by Rockafellar [3,4] is one of the classical methods for solving zero points of maximal monotone operators. In this paper, we investigate the problem of finding a zero of the sum of two monotone operators. The problem is very general in the sense that it includes, as special cases, convexly constrained linear inverse problems, split feasibility problem, convexly constrained minimization problems, fixed point problems, variational inequalities, Nash equilibrium problem in noncooperative games and others. Because of their importance, splitting methods, which were proposed by Lions and Mercier [5] and Passty [6], for zero problems have been studied extensively recently; see, for instance, [7-17] and the references therein.

Let $C$ be a nonempty closed and convex subset of $H$. Let $A: C \rightarrow H$ be a mapping. Recall that the classical variational inequality problem is to find a point $x \in C$ such that

$$
\langle y-x, A x\rangle \geq 0, \quad \forall y \in C
$$

Such a point $x \in C$ is called a solution of variational inequality (1.1). In this paper, we use $V I(C, A)$ to denote the solution set of variational inequality (1.1). Recall that $A$ is said to be

○2015 Zhang; licensee Springer. This is an Open Access article distributed under the terms of the Creative Commons Attribution License (http://creativecommons.org/licenses/by/2.0), which permits unrestricted use, distribution, and reproduction in any medium, provided the original work is properly cited. 
monotone iff

$$
\langle A x-A y, x-y\rangle \geq 0, \quad \forall x, y \in C
$$

Recall that $A$ is said to be inverse-strongly monotone iff there exists a constant $\kappa>0$ such that

$$
\langle A x-A y, x-y\rangle \geq \kappa\|A x-A y\|^{2}, \quad \forall x, y \in C .
$$

For such a case, we also call $A$ is $\kappa$-inverse-strongly monotone. It is also not hard to see that every inverse-strongly monotone mapping is monotone and continuous.

Let $S: C \rightarrow C$ be a mapping. In this paper, we use $F(S)$ to denote the fixed point set of $S$. $S$ is said to be contractive iff there exists a constant $\beta \in(0,1)$ such that

$$
\|S x-S y\| \leq \beta\|x-y\|, \quad \forall x, y \in C
$$

We also call $S$ is $\beta$-contractive. $S$ is said to be nonexpansive iff

$$
\|S x-S y\| \leq\|x-y\|, \quad \forall x, y \in C .
$$

It is well known if $C$ is nonempty closed convex of $H$, then $F(S)$ is not empty. $S$ is said to be firmly nonexpansive iff

$$
\|S x-S y\|^{2} \leq\|x-y\|^{2}-\|(I-S) x-(I-S) y\|^{2}, \quad \forall x, y \in C .
$$

In order to prove our main results, we also need the following lemmas.

Lemma 1.1 [18] Let $A$ be a maximal monotone operator on $H$. For $\lambda>0, \mu>0$, and $x \in E$, we have $J_{\lambda} x=J_{\mu}\left(\frac{\mu}{\lambda} x+\left(1-\frac{\mu}{\lambda}\right) J_{\lambda} x\right)$, where $J_{\lambda}=(I+\lambda A)^{-1}$ and $J_{\mu}=(I+\mu A)^{-1}$.

Lemma 1.2 [19] Let $\left\{x_{n}\right\}$ and $\left\{y_{n}\right\}$ be bounded sequences in H. Let $\left\{\beta_{n}\right\}$ be a sequence in $(0,1)$ with $0<\liminf _{n \rightarrow \infty} \beta_{n} \leq \limsup _{n \rightarrow \infty} \beta_{n}<1$. Suppose that $x_{n+1}=\left(1-\beta_{n}\right) y_{n}+\beta_{n} x_{n}$, $\forall n \geq 1$ and

$$
\limsup _{n \rightarrow \infty}\left(\left\|y_{n+1}-y_{n}\right\|-\left\|x_{n+1}-x_{n}\right\|\right) \leq 0
$$

Then $\lim _{n \rightarrow \infty}\left\|y_{n}-x_{n}\right\|=0$.

Lemma 1.3 [20] Let $\left\{a_{n}\right\}$ be a sequence of nonnegative numbers satisfying the condition $a_{n+1} \leq\left(1-t_{n}\right) a_{n}+t_{n} b_{n}, \forall n \geq 0$, where $\left\{t_{n}\right\}$ is a number sequence in $(0,1)$ such that $\lim _{n \rightarrow \infty} t_{n}=0$ and $\sum_{n=0}^{\infty} t_{n}=\infty,\left\{b_{n}\right\}$ is a number sequence such that $\lim _{\sup _{n \rightarrow \infty}} b_{n} \leq 0$. Then $\lim _{n \rightarrow \infty} a_{n}=0$.

Lemma 1.4 [21] Let $C$ be a nonempty closed convex subset of $H$. Let $A: C \rightarrow H$ be a mapping and let $B: H \rightrightarrows H$ be a maximal monotone operator. Then $\left\|x-(I+s B)^{-1} x\right\| \leq$ $2\left\|x-(I+r B)^{-1} x\right\|$ for all $0<s \leq r$. 
Lemma 1.5 [22] Let $\left\{\lambda_{n}\right\}$ be a real sequence that does not decreasing at infinity, in the sense that there exists a subsequence $\left\{\lambda_{n_{k}}\right\}$ such that $\lambda_{n_{k}} \leq \lambda_{n_{k}+1}$ for all $k \geq 0$. For every $n>n_{0}$, define an integer sequence $d(n)$ as $d(n)=\max \left\{n_{0} \leq k \leq n \mid \lambda_{n_{k}} \leq \lambda_{n_{k}+1}\right\}$. Then $\lim _{n \rightarrow \infty} d(n)=$ 0 and for all $n>n_{0} \max \left\{\lambda_{d(n)}, \lambda_{n}\right\} \leq \lambda_{d(n)+1}$.

Lemma 1.6 [23] Let $C$ be a nonempty closed convex subset of $H$. Let $S: C \rightarrow C$ be a nonexpansive mapping with a nonempty fixed point set. If $\left\{x_{n}\right\}$ converges weakly to $x$ and $\left\{\left\|x_{n}-T x_{n}\right\|\right\}$ converges to zero. Then $x \in F(S)$.

\section{Main results}

Now, we are in a position to state our main results.

Theorem 2.1 Let $C$ be a nonempty closed convex subset of $H$. Let $S: C \rightarrow C$ be a nonexpansive mapping with fixed points and let $f: C \rightarrow C$ be a $\beta$-contractive mapping. Let $A: C \rightarrow H$ be an $\alpha$-inverse-strongly monotone mapping and let $B$ be a maximal monotone operator on $H$. Assume that $\operatorname{Dom}(B) \subset C$ and $F(S) \cap(A+B)^{-1}(0)$ is not empty. Let $\left\{\alpha_{n}\right\}$ and $\left\{\beta_{n}\right\}$ be real number sequences in $(0,1)$ and let $\left\{r_{n}\right\}$ be a positive real number sequence in $(0,2 \alpha)$. Let $\left\{x_{n}\right\}$ be a sequence generated in the following process: $x_{1} \in C$ and

$$
\left\{\begin{array}{l}
y_{n}=\alpha_{n} f\left(x_{n}\right)+\left(1-\alpha_{n}\right) x_{n}, \\
x_{n+1}=\beta_{n} x_{n}+\left(1-\beta_{n}\right) S\left(I+r_{n} B\right)^{-1}\left(y_{n}-r_{n} A y_{n}\right), \quad \forall n \geq 1 .
\end{array}\right.
$$

Assume that the control sequences satisfy the following restrictions:

(a) $\lim _{n \rightarrow \infty} \alpha_{n}=0, \sum_{n=0}^{\infty} \alpha_{n}=\infty$;

(b) $0<\liminf _{n \rightarrow \infty} \beta_{n} \leq \limsup _{n \rightarrow \infty} \beta_{n}<1$;

(c) $0<a \leq r_{n} \leq b<2 \alpha$ and $\sum_{n=1}^{\infty}\left|r_{n}-r_{n-1}\right|<\infty$,

where $a$ and $b$ are two real numbers. Then $\left\{x_{n}\right\}$ converges strongly to a point $\bar{x} \in F(S) \cap(A+$ $B)^{-1}(0)$, which is also a unique solution to the following variational inequality:

$$
\langle f(\bar{x})-\bar{x}, p-\bar{x}\rangle \leq 0, \quad \forall p \in F(S) \cap(A+B)^{-1}(0) .
$$

Proof Note that the mapping $I-r_{n} A$ is nonexpansive. Indeed, we have

$$
\begin{aligned}
\left\|\left(I-r_{n} A\right) x-\left(I-r_{n} A\right) y\right\|^{2} \\
\quad=\|x-y\|^{2}-2 r_{n}\langle x-y, A x-A y\rangle+r_{n}{ }^{2}\|A x-A y\|^{2} \\
\quad \leq\|x-y\|^{2}-r_{n}\left(2 \alpha-r_{n}\right)\|A x-A y\|^{2} .
\end{aligned}
$$

In light of restriction (c), one finds that $I-r_{n} A$ is nonexpansive. It is obvious that $F((I+$ $\left.\left.r_{n} B\right)^{-1}\left(I-r_{n} A\right)\right)=(A+B)^{-1}(0)$. Fix $p \in(A+B)^{-1}(0) \cap F(S)$. It follows that

$$
\begin{aligned}
\left\|y_{n}-p\right\| & \leq \alpha_{n}\left\|f\left(x_{n}\right)-p\right\|+\left(1-\alpha_{n}\right)\left\|x_{n}-p\right\| \\
& \leq\left(1-\alpha_{n}(1-\beta)\right)\left\|x_{n}-p\right\|+\alpha_{n}\|f(p)-p\| .
\end{aligned}
$$


Putting $J_{r_{n}}=\left(I+r_{n} B\right)^{-1}$, we see that

$$
\begin{aligned}
\left\|x_{n+1}-p\right\| & \leq \beta_{n}\left\|x_{n}-p\right\|+\left(1-\beta_{n}\right)\left\|S J_{r_{n}}\left(y_{n}-r_{n} A y_{n}\right)-p\right\| \\
& \leq \beta_{n}\left\|x_{n}-p\right\|+\left(1-\beta_{n}\right)\left\|\left(y_{n}-r_{n} A y_{n}\right)-p\right\| \\
& \leq\left(1-\alpha_{n}\left(1-\beta_{n}\right)(1-\beta)\right)\left\|x_{n}-p\right\|+\alpha_{n}\left(1-\beta_{n}\right)(1-\beta) \frac{\|f(p)-p\|}{1-\beta} .
\end{aligned}
$$

By mathematical induction, we find that the sequence $\left\{x_{n}\right\}$ is bounded. Note that

$$
\begin{aligned}
\left\|y_{n}-y_{n-1}\right\| \leq & \alpha_{n}\left\|f\left(x_{n}\right)-f\left(x_{n-1}\right)\right\|+\left(1-\alpha_{n}\right)\left\|x_{n}-x_{n-1}\right\| \\
& +\left|\alpha_{n}-\alpha_{n-1}\right|\left\|f\left(x_{n-1}\right)-x_{n-1}\right\| \\
\leq & \left(1-\alpha_{n}(1-\beta)\right)\left\|x_{n}-x_{n-1}\right\|+\left|\alpha_{n}-\alpha_{n-1}\right|\left\|f\left(x_{n-1}\right)-x_{n-1}\right\| .
\end{aligned}
$$

Putting $z_{n}=y_{n}-r_{n} A y_{n}$, we find from Lemma 1.1 that

$$
\begin{aligned}
\left\|J_{r_{n}} z_{n}-J_{r_{n-1}} z_{n-1}\right\| \leq & \left\|\frac{r_{n-1}}{r_{n}}\left(z_{n}-z_{n-1}\right)+\left(1-\frac{r_{n-1}}{r_{n}}\right)\left(J_{r_{n}} z_{n}-z_{n-1}\right)\right\| \\
\leq & \left\|z_{n}-z_{n-1}\right\|+\frac{\left|r_{n}-r_{n-1}\right|}{a}\left\|J_{r_{n}} z_{n}-z_{n}\right\| \\
\leq & \left\|y_{n}-y_{n-1}\right\|+\left|r_{n}-r_{n-1}\right|\left\|A y_{n-1}\right\|+\frac{\left|r_{n}-r_{n-1}\right|}{a}\left\|J_{r_{n}} z_{n}-z_{n}\right\| \\
\leq & \left(1-\alpha_{n}(1-\beta)\right)\left\|x_{n}-x_{n-1}\right\|+\left|\alpha_{n}-\alpha_{n-1}\right|\left\|f\left(x_{n-1}\right)-x_{n-1}\right\| \\
& \quad+\left|r_{n}-r_{n-1}\right|\left\|A y_{n-1}\right\|+\frac{\left|r_{n}-r_{n-1}\right|}{a}\left\|J_{r_{n}} z_{n}-z_{n}\right\| .
\end{aligned}
$$

This yields

$$
\begin{aligned}
\left\|S J_{r_{n}} z_{n}-S J_{r_{n-1}} z_{n-1}\right\| \leq & \left\|J_{r_{n}} z_{n}-J_{r_{n-1}} z_{n-1}\right\| \\
\leq & \left\|x_{n}-x_{n-1}\right\|+\left|\alpha_{n}-\alpha_{n-1}\right|\left\|f\left(x_{n-1}\right)-x_{n-1}\right\| \\
& +\left|r_{n}-r_{n-1}\right|\left\|A y_{n-1}\right\|+\frac{\left|r_{n}-r_{n-1}\right|}{a}\left\|J_{r_{n}} z_{n}-z_{n}\right\| .
\end{aligned}
$$

It follows from restrictions (a) and (c) that

$$
\limsup _{n \rightarrow \infty}\left(\left\|S J_{r_{n}} z_{n}-S J_{r_{n-1}} z_{n-1}\right\|-\left\|x_{n}-x_{n-1}\right\|\right) \leq 0
$$

Using Lemma 1.2, we have $\lim _{n \rightarrow \infty}\left\|S J_{r_{n}} z_{n}-x_{n}\right\|=0$. It follows that

$$
\lim _{n \rightarrow \infty}\left\|x_{n+1}-x_{n}\right\|=0
$$

Since $\left\|y_{n}-x_{n}\right\|=\alpha_{n}\left\|f\left(x_{n}\right)-x_{n}\right\|$, we find that

$$
\lim _{n \rightarrow \infty}\left\|y_{n}-x_{n}\right\|=0
$$


Since $\|\cdot\|^{2}$ is convex, we find that

$$
\left\|y_{n}-p\right\|^{2} \leq \alpha_{n}\left\|f\left(x_{n}\right)-p\right\|^{2}+\left(1-\alpha_{n}\right)\left\|x_{n}-p\right\|^{2} .
$$

It follows that

$$
\begin{aligned}
\left\|x_{n+1}-p\right\|^{2} & \leq \beta_{n}\left\|x_{n}-p\right\|^{2}+\left(1-\beta_{n}\right)\left\|S J_{r_{n}} z_{n}-p\right\|^{2} \\
& \leq \beta_{n}\left\|x_{n}-p\right\|^{2}+\left(1-\beta_{n}\right)\left\|J_{r_{n}}\left(I-r_{n} A\right) y_{n}-p\right\|^{2} \\
& \leq \beta_{n}\left\|x_{n}-p\right\|^{2}+\left(1-\beta_{n}\right)\left\|\left(I-r_{n} A\right) y_{n}-p\right\|^{2} \\
& \leq \beta_{n}\left\|x_{n}-p\right\|^{2}+\left(1-\beta_{n}\right)\left\|y_{n}-p\right\|^{2}-r_{n}\left(1-\beta_{n}\right)\left(2 \alpha-r_{n}\right)\left\|A y_{n}-A p\right\|^{2} \\
& \leq\left\|x_{n}-p\right\|^{2}+\alpha_{n}\left\|f\left(x_{n}\right)-p\right\|^{2}-r_{n}\left(1-\beta_{n}\right)\left(2 \alpha-r_{n}\right)\left\|A y_{n}-A p\right\|^{2} .
\end{aligned}
$$

Hence, we have

$$
\begin{aligned}
& r_{n}\left(1-\beta_{n}\right)\left(2 \alpha-r_{n}\right)\left\|A y_{n}-A p\right\|^{2} \\
& \quad \leq\left(\left\|x_{n}-p\right\|+\left\|x_{n+1}-p\right\|\right)\left\|x_{n+1}-x_{n}\right\|+\alpha_{n}\left\|f\left(x_{n}\right)-p\right\|^{2} .
\end{aligned}
$$

In view of restrictions (a), (b), and (c), we find from (2.1) that

$$
\lim _{n \rightarrow \infty}\left\|A y_{n}-A p\right\|=0
$$

Since $J_{r_{n}}$ is firmly nonexpansive, we have

$$
\begin{aligned}
\left\|J_{r_{n}} z_{n}-p\right\|^{2} \leq & \left\langle J_{r_{n}} z_{n}-p,\left(y_{n}-r_{n} A y_{n}\right)-\left(p-r_{n} A p\right)\right\rangle \\
= & \frac{1}{2}\left(\left\|J_{r_{n}} z_{n}-p\right\|^{2}+\left\|\left(y_{n}-r_{n} A y_{n}\right)-\left(p-r_{n} A p\right)\right\|^{2}\right. \\
& \left.-\left\|\left(J_{r_{n}} z_{n}-p\right)-\left(\left(y_{n}-r_{n} A y_{n}\right)-\left(p-r_{n} A p\right)\right)\right\|^{2}\right) \\
\leq & \frac{1}{2}\left(\left\|J_{r_{n}} z_{n}-p\right\|^{2}+\left\|y_{n}-p\right\|^{2}-\left\|J_{r_{n}} z_{n}-y_{n}\right\|^{2}\right. \\
& \left.+2 r_{n}\left\|A y_{n}-A p\right\|\left\|J_{r_{n}} z_{n}-y_{n}\right\|\right) .
\end{aligned}
$$

This implies from (2.3) that

$$
\begin{gathered}
\left\|J_{r_{n}} z_{n}-p\right\|^{2} \leq \\
\leq y_{n}-p\left\|^{2}-\right\| J_{r_{n}} z_{n}-y_{n}\left\|^{2}+2 r_{n}\right\| A y_{n}-A p\|\| J_{r_{n}} z_{n}-y_{n} \| \\
\leq \alpha_{n}\left\|f\left(x_{n}\right)-p\right\|^{2}+\left(1-\alpha_{n}\right)\left\|x_{n}-p\right\|^{2}-\left\|J_{r_{n}} z_{n}-y_{n}\right\|^{2} \\
+2 r_{n}\left\|A y_{n}-A p\right\|\left\|J_{r_{n}} z_{n}-y_{n}\right\| .
\end{gathered}
$$

On the other hand, we have

$$
\begin{aligned}
\left\|x_{n+1}-p\right\|^{2} & \leq \beta_{n}\left\|x_{n}-p\right\|^{2}+\left(1-\beta_{n}\right)\left\|S J_{r_{n}} z_{n}-p\right\|^{2} \\
& \leq \beta_{n}\left\|x_{n}-p\right\|^{2}+\left(1-\beta_{n}\right)\left\|J_{r_{n}} z_{n}-p\right\|^{2}
\end{aligned}
$$




$$
\begin{aligned}
\leq & \left\|x_{n}-p\right\|^{2}+\alpha_{n}\left\|f\left(x_{n}\right)-p\right\|^{2}-\left(1-\beta_{n}\right)\left\|J_{r_{n}} z_{n}-y_{n}\right\|^{2} \\
& +2 r_{n}\left\|A y_{n}-A p\right\|\left\|J_{r_{n}} z_{n}-y_{n}\right\| .
\end{aligned}
$$

This implies that

$$
\begin{aligned}
\left(1-\beta_{n}\right)\left\|J_{r_{n}} z_{n}-y_{n}\right\|^{2} \leq & \left(\left\|x_{n}-p\right\|+\left\|x_{n+1}-p\right\|\right)\left\|x_{n}-x_{n+1}\right\|+\alpha_{n}\left\|f\left(x_{n}\right)-p\right\|^{2} \\
& +2 r_{n}\left\|A y_{n}-A p\right\|\left\|J_{r_{n}} z_{n}-y_{n}\right\| .
\end{aligned}
$$

In view of restrictions (a) and (b), we find from (2.1) and (2.4) that

$$
\lim _{n \rightarrow \infty}\left\|J_{r_{n}} z_{n}-y_{n}\right\|=0
$$

Next, we show that $\lim \sup _{n \rightarrow \infty}\left\langle f(\bar{x})-\bar{x}, y_{n}-\bar{x}\right\rangle \leq 0$, where $\bar{x}=\operatorname{Proj}_{F(S) \cap(A+B)^{-1}(0)} f(\bar{x})$. To show it, we can choose a subsequence $\left\{y_{n_{i}}\right\}$ of $\left\{y_{n}\right\}$ such that

$$
\limsup _{n \rightarrow \infty}\left\langle f(\bar{x})-\bar{x}, y_{n}-\bar{x}\right\rangle=\lim _{i \rightarrow \infty}\left\langle f(\bar{x})-\bar{x}, y_{n_{i}}-\bar{x}\right\rangle .
$$

Since $\left\{y_{n_{i}}\right\}$ is bounded, we can choose a subsequence $\left\{y_{n_{i}}\right\}$ of $\left\{y_{n_{i}}\right\}$ which converges weakly some point $x$. We may assume, without loss of generality, that $y_{n_{i}}$ converges weakly to $x$.

Now, we are in a position to show that $x \in(A+B)^{-1}(0)$. Set $\lambda_{n}=J_{r_{n}}\left(y_{n}-r_{n} A y_{n}\right)$. It follows that $\frac{y_{n}-\lambda_{n}}{r_{n}}-A y_{n} \in B \lambda_{n}$. Since $B$ is monotone, we get, for any $(\mu, \nu) \in B$,

$$
\left\langle\lambda_{n}-\mu, \frac{y_{n}-\lambda_{n}}{r_{n}}-A y_{n}-v\right\rangle \geq 0
$$

Replacing $n$ by $n_{i}$ and letting $i \rightarrow \infty$, we obtain from (2.5) that

$$
\langle x-\mu,-A x-v\rangle \geq 0
$$

This gives $-A x \in B x$, that is, $0 \in(A+B)(x)$. This proves that $x \in(A+B)^{-1}(0)$.

Now, we are in a position to prove that $x \in F(S)$. Notice that

$$
\left\|S \lambda_{n}-y_{n}\right\| \leq \frac{1}{1-\beta_{n}}\left\|x_{n+1}-y_{n}\right\|+\frac{\beta_{n}}{1-\beta_{n}}\left\|y_{n}-x_{n}\right\| .
$$

This implies that $\lim _{n \rightarrow \infty}\left\|S \lambda_{n}-y_{n}\right\|=0$. This implies from (2.5) that $\left\|S \lambda_{n}-\lambda_{n}\right\| \rightarrow 0$. Since $I-S$ is demiclosed at zero, we find that $x \in F(S)$. This complete the proof that $x \in$ $F(S) \cap(A+B)^{-1}(0)$. It follows that

$$
\limsup _{n \rightarrow \infty}\left\langle f(\bar{x})-\bar{x}, y_{n}-\bar{x}\right\rangle \leq 0
$$

Finally, we show that $x_{n} \rightarrow \bar{x}$. Notice that

$$
\begin{aligned}
\left\|y_{n}-\bar{x}\right\|^{2} & \leq \alpha_{n}\left\langle f\left(x_{n}\right)-\bar{x}, y_{n}-\bar{x}\right\rangle+\left(1-\alpha_{n}\right)\left\|x_{n}-\bar{x}\right\|\left\|y_{n}-\bar{x}\right\| \\
& \leq\left(1-\alpha_{n}(1-\beta)\right)\left\|x_{n}-\bar{x}\right\|\left\|y_{n}-\bar{x}\right\|+\alpha_{n}\left\langle f(\bar{x})-\bar{x}, y_{n}-\bar{x}\right\rangle .
\end{aligned}
$$


This implies that

$$
\left\|y_{n}-\bar{x}\right\|^{2} \leq 2 \alpha_{n}\left\langle f(\bar{x})-\bar{x}, y_{n}-\bar{x}\right\rangle+\left(1-\alpha_{n}(1-\beta)\right)\left\|x_{n}-\bar{x}\right\|^{2} .
$$

It follows that

$$
\begin{aligned}
\left\|x_{n+1}-\bar{x}\right\|^{2} & \leq \beta_{n}\left\|x_{n}-\bar{x}\right\|^{2}+\left(1-\beta_{n}\right)\left\|S J_{r_{n}}\left(I-r_{n} A\right) y_{n}-\bar{x}\right\|^{2} \\
& \leq \beta_{n}\left\|x_{n}-\bar{x}\right\|^{2}+\left(1-\beta_{n}\right)\left\|y_{n}-\bar{x}\right\|^{2} \\
& \leq\left(1-\alpha_{n}\left(1-\beta_{n}\right)(1-\beta)\right)\left\|x_{n}-\bar{x}\right\|^{2}+2 \alpha_{n}\left(1-\beta_{n}\right)\left\langle f(\bar{x})-\bar{x}, y_{n}-\bar{x}\right\rangle .
\end{aligned}
$$

In view of restrictions (a) and (b), we find from Lemma 1.3 that $x_{n} \rightarrow \bar{x}$. This completes the proof.

From Theorem 2.1, we have the following results immediately.

Corollary 2.2 Let $C$ be a nonempty closed convex subset of $H$. Let $S: C \rightarrow C$ be a nonexpansive mapping with fixed points and let $f: C \rightarrow C$ be a $\beta$-contractive mapping. Let $\left\{\alpha_{n}\right\}$ and $\left\{\beta_{n}\right\}$ be real number sequences in $(0,1)$. Let $\left\{x_{n}\right\}$ be a sequence generated in the following process: $x_{1} \in C$ and

$$
\left\{\begin{array}{l}
y_{n}=\alpha_{n} f\left(x_{n}\right)+\left(1-\alpha_{n}\right) x_{n}, \\
x_{n+1}=\beta_{n} x_{n}+\left(1-\beta_{n}\right) S y_{n}, \quad \forall n \geq 1
\end{array}\right.
$$

Assume that the control sequences satisfy the following restrictions:

(a) $\lim _{n \rightarrow \infty} \alpha_{n}=0, \sum_{n=0}^{\infty} \alpha_{n}=\infty$;

(b) $0<\liminf _{n \rightarrow \infty} \beta_{n} \leq \limsup _{n \rightarrow \infty} \beta_{n}<1$.

Then $\left\{x_{n}\right\}$ converges strongly to a point $\bar{x} \in F(S)$, which is also a unique solution to the following variational inequality:

$$
\langle f(\bar{x})-\bar{x}, p-\bar{x}\rangle \leq 0, \quad \forall p \in F(S)
$$

Corollary 2.3 Let $C$ be a nonempty closed convex subset of $H$. Let $f: C \rightarrow C$ be a $\beta$ contractive mapping. Let $A: C \rightarrow H$ be an $\alpha$-inverse-strongly monotone mapping and let $B$ be a maximal monotone operator on $H$. Assume that $\operatorname{Dom}(B) \subset C$ and $(A+B)^{-1}(0)$ is not empty. Let $\left\{\alpha_{n}\right\}$ and $\left\{\beta_{n}\right\}$ be real number sequences in $(0,1)$ and $\left\{r_{n}\right\}$ be a positive real number sequence in $(0,2 \alpha)$. Let $\left\{x_{n}\right\}$ be a sequence generated in the following process: $x_{1} \in C$ and

$$
\left\{\begin{array}{l}
y_{n}=\alpha_{n} f\left(x_{n}\right)+\left(1-\alpha_{n}\right) x_{n}, \\
x_{n+1}=\beta_{n} x_{n}+\left(1-\beta_{n}\right)\left(I+r_{n} B\right)^{-1}\left(y_{n}-r_{n} A y_{n}\right), \quad \forall n \geq 1 .
\end{array}\right.
$$

Assume that the control sequences satisfy the following restrictions:

(a) $\lim _{n \rightarrow \infty} \alpha_{n}=0, \sum_{n=0}^{\infty} \alpha_{n}=\infty$;

(b) $0<\liminf _{n \rightarrow \infty} \beta_{n} \leq \limsup _{n \rightarrow \infty} \beta_{n}<1$; 
(c) $0<a \leq r_{n} \leq b<2 \alpha$ and $\sum_{n=1}^{\infty}\left|r_{n}-r_{n-1}\right|<\infty$,

where $a$ and $b$ are two real numbers. Then $\left\{x_{n}\right\}$ converges strongly to a point $\bar{x} \in(A+B)^{-1}(0)$, which is also a unique solution to the following variational inequality:

$$
\langle f(\bar{x})-\bar{x}, p-\bar{x}\rangle \leq 0, \quad \forall p \in(A+B)^{-1}(0) .
$$

Next, we give a result on the zeros of the sum of the operators $A$ and $B$ based on a different method.

Theorem 2.4 Let $C$ be a nonempty closed convex subset of $H$. Let $f: C \rightarrow C$ be a $\beta$ contractive mapping. Let $A: C \rightarrow H$ be an $\alpha$-inverse-strongly monotone mapping and let $B$ be a maximal monotone operator on $H$. Assume that $\operatorname{Dom}(B) \subset C$ and $(A+B)^{-1}(0)$ is not empty. Let $\left\{\alpha_{n}\right\}$ and $\left\{\beta_{n}\right\}$ be real number sequences in $(0,1)$ and $\left\{r_{n}\right\}$ be a positive real number sequence in $(0,2 \alpha)$. Let $\left\{x_{n}\right\}$ be a sequence generated in the following process: $x_{1} \in C$ and

$$
\left\{\begin{array}{l}
y_{n}=\alpha_{n} f\left(x_{n}\right)+\left(1-\alpha_{n}\right) x_{n}, \\
x_{n+1}=\beta_{n} x_{n}+\left(1-\beta_{n}\right)\left(I+r_{n} B\right)^{-1}\left(y_{n}-r_{n} A y_{n}\right), \quad \forall n \geq 1 .
\end{array}\right.
$$

Assume that the control sequences satisfy the following restrictions:

(a) $\lim _{n \rightarrow \infty} \alpha_{n}=0, \sum_{n=0}^{\infty} \alpha_{n}=\infty$;

(b) $0 \leq \beta_{n} \leq \bar{\beta}<1$;

(c) $0<a \leq r_{n} \leq b<2 \alpha$,

where $\bar{\beta}, a$, and $b$ are real numbers. Then $\left\{x_{n}\right\}$ converges strongly to a point $\bar{x} \in(A+B)^{-1}(0)$, which is also a unique solution to the following variational inequality:

$$
\langle f(\bar{x})-\bar{x}, p-\bar{x}\rangle \leq 0, \quad \forall p \in(A+B)^{-1}(0) .
$$

Proof From the proof of Theorem 2.1, we find that $\left\{x_{n}\right\}$ is bounded. Since $P_{F(S) \cap(A+B)^{-1}(0)} f$ is contractive, it has a unique fixed point. Next, we use $\bar{x}$ to denote the unique fixed point. Note that

$$
\begin{aligned}
\left\|y_{n}-\bar{x}\right\|^{2} & =\alpha_{n}\left\langle f\left(x_{n}\right)-\bar{x}, y_{n}-\bar{x}\right\rangle+\left(1-\alpha_{n}\right)\left\langle x_{n}-\bar{x}, y_{n}-\bar{x}\right\rangle \\
& \leq\left(1-\alpha_{n}(1-\beta)\right)\left\|x_{n}-\bar{x}\right\|\left\|y_{n}-\bar{x}\right\|+\alpha_{n}\left\langle f(\bar{x})-\bar{x}, y_{n}-\bar{x}\right\rangle \\
& \leq \frac{\left(1-\alpha_{n}(1-\beta)\right.}{2}\left(\left\|x_{n}-\bar{x}\right\|^{2}+\left\|y_{n}-\bar{x}\right\|^{2}\right)+\alpha_{n}\left\langle f(\bar{x})-\bar{x}, y_{n}-\bar{x}\right\rangle .
\end{aligned}
$$

It follows that

$$
\left\|y_{n}-\bar{x}\right\|^{2} \leq\left(1-\frac{2 \alpha_{n}(1-\beta)}{1+\alpha_{n}(1-\beta)}\right)\left\|x_{n}-\bar{x}\right\|^{2}+\frac{2 \alpha_{n}}{1+\alpha_{n}(1-\beta)}\left\langle f(\bar{x})-\bar{x}, y_{n}-\bar{x}\right\rangle .
$$

Since $J_{r_{n}}$ is firmly nonexpansive and $A$ is inverse-strongly monotone, we find that

$$
\begin{aligned}
\left\|J_{r_{n}}\left(y_{n}-r_{n} A y_{n}\right)-\bar{x}\right\|^{2} \leq & \left\|\left(y_{n}-r_{n} A y_{n}\right)-\left(\bar{x}-r_{n} A \bar{x}\right)\right\|^{2} \\
& -\left\|\left(I-J_{r_{n}}\right)\left(y_{n}-r_{n} A y_{n}\right)-\left(I-J_{r_{n}}\right)\left(\bar{x}-r_{n} A \bar{x}\right)\right\|^{2}
\end{aligned}
$$




$$
\begin{aligned}
\leq & \left\|y_{n}-\bar{x}\right\|^{2}-r_{n}\left(2 \alpha-r_{n}\right)\left\|A y_{n}-A \bar{x}\right\|^{2} \\
& -\left\|\left(I-J_{r_{n}}\right)\left(y_{n}-r_{n} A y_{n}\right)-\left(I-J_{r_{n}}\right)\left(\bar{x}-r_{n} A \bar{x}\right)\right\|^{2} .
\end{aligned}
$$

Substituting (2.6) into (2.7), we find that

$$
\begin{aligned}
& \left\|J_{r_{n}}\left(y_{n}-r_{n} A y_{n}\right)-\bar{x}\right\|^{2} \\
& \quad \leq\left(1-\frac{2 \alpha_{n}(1-\beta)}{1+\alpha_{n}(1-\beta)}\right)\left\|x_{n}-\bar{x}\right\|^{2}+\frac{2 \alpha_{n}}{1+\alpha_{n}(1-\beta)}\left\langle f(\bar{x})-\bar{x}, y_{n}-\bar{x}\right\rangle \\
& \quad-r_{n}\left(2 \alpha-r_{n}\right)\left\|A y_{n}-A \bar{x}\right\|^{2}-\left\|\left(I-J_{r_{n}}\right)\left(y_{n}-r_{n} A y_{n}\right)-\left(I-J_{r_{n}}\right)\left(\bar{x}-r_{n} A \bar{x}\right)\right\|^{2} .
\end{aligned}
$$

It follows that

$$
\begin{aligned}
\left\|x_{n+1}-\bar{x}\right\|^{2} \leq & \beta_{n}\left\|x_{n}-\bar{x}\right\|^{2}+\left(1-\beta_{n}\right)\left\|J_{r_{n}}\left(y_{n}-r_{n} A y_{n}\right)-\bar{x}\right\|^{2} \\
\leq & \left(1-\frac{2 \alpha_{n}\left(1-\beta_{n}\right)(1-\beta)}{1+\alpha_{n}(1-\beta)}\right)\left\|x_{n}-\bar{x}\right\|^{2}+\frac{2 \alpha_{n}\left(1-\beta_{n}\right)}{1+\alpha_{n}(1-\beta)}\left\langle f(\bar{x})-\bar{x}, y_{n}-\bar{x}\right\rangle \\
& -\left(1-\beta_{n}\right) r_{n}\left(2 \alpha-r_{n}\right)\left\|A y_{n}-A \bar{x}\right\|^{2} \\
& -\left(1-\beta_{n}\right)\left\|\left(I-J_{r_{n}}\right)\left(y_{n}-r_{n} A y_{n}\right)-\left(I-J_{r_{n}}\right)\left(\bar{x}-r_{n} A \bar{x}\right)\right\|^{2}
\end{aligned}
$$

Next, we consider the following possible two cases.

Case 1. Suppose that there exists some nonnegative integer $m$ such that the sequence $\left\{\left\|x_{n}-\bar{x}\right\|\right\}$ is eventually decreasing. Then $\lim _{n \rightarrow \infty}\left\|x_{n}-\bar{x}\right\|$ exists. By (2.8), we find that

$$
\begin{aligned}
& \left(1-\beta_{n}\right) r_{n}\left(2 \alpha-r_{n}\right)\left\|A y_{n}-A \bar{x}\right\|^{2} \\
& \quad \leq\left\|x_{n}-\bar{x}\right\|^{2}-\left\|x_{n+1}-\bar{x}\right\|^{2}+2 \alpha_{n}\|f(\bar{x})-\bar{x}\|\left\|y_{n}-\bar{x}\right\| .
\end{aligned}
$$

By use of restrictions (b) and (c), we have $\lim _{n \rightarrow \infty}\left\|A y_{n}-A \bar{x}\right\|=0$. It also follows from (2.8) that

$$
\begin{aligned}
& \left(1-\beta_{n}\right)\left\|\left(I-J_{r_{n}}\right)\left(y_{n}-r_{n} A y_{n}\right)-\left(I-J_{r_{n}}\right)\left(\bar{x}-r_{n} A \bar{x}\right)\right\|^{2} \\
& \quad \leq\left\|x_{n}-\bar{x}\right\|^{2}-\left\|x_{n+1}-\bar{x}\right\|^{2}+2 \alpha_{n}\|f(\bar{x})-\bar{x}\|\left\|y_{n}-\bar{x}\right\| .
\end{aligned}
$$

From restrictions (b) and (c), we obtain

$$
\lim _{n \rightarrow \infty}\left\|\left(I-J_{r_{n}}\right)\left(y_{n}-r_{n} A y_{n}\right)-\left(I-J_{r_{n}}\right)\left(\bar{x}-r_{n} A \bar{x}\right)\right\|=0 .
$$

Hence, we have $\lim _{n \rightarrow \infty}\left\|y_{n}-J_{r_{n}}\left(y_{n}-r_{n} A y_{n}\right)\right\|=0$. From Lemma 1.4, we find that $\| y_{n}-$ $J_{r}\left(y_{n}-r A y_{n}\right)\|\leq 2\| y_{n}-J_{r_{n}}\left(y_{n}-r_{n} A y_{n}\right) \|$. This implies that $\lim _{n \rightarrow \infty}\left\|y_{n}-J_{r}\left(y_{n}-r A y_{n}\right)\right\|=0$. Next, we show that $\limsup _{n \rightarrow \infty}\left\langle f(\bar{x})-\bar{x}, y_{n}-\bar{x}\right\rangle \leq 0$. To show it, we can choose a subsequence $\left\{y_{n_{i}}\right\}$ of $\left\{y_{n}\right\}$ such that

$$
\limsup _{n \rightarrow \infty}\left\langle f(\bar{x})-\bar{x}, y_{n}-\bar{x}\right\rangle=\lim _{i \rightarrow \infty}\left\langle f(\bar{x})-\bar{x}, y_{n_{i}}-\bar{x}\right\rangle .
$$

Since $\left\{y_{n_{i}}\right\}$ is bounded, we can choose a subsequence $\left\{y_{n_{i}}\right\}$ of $\left\{y_{n_{i}}\right\}$ which converges weakly to some point $x$. We may assume, without loss of generality, that $y_{n_{i}}$ converges weakly to $x$. 
Since the mapping $J_{r}(I-r A)$ is nonexpansive, we find that $x \in F\left(J_{r}(I-r A)\right)=(A+B)^{-1}(0)$. It follows that $\lim _{\sup _{n \rightarrow \infty}}\left\langle f(\bar{x})-\bar{x}, y_{n}-\bar{x}\right\rangle \leq 0$. In view of (2.8), we find from Lemma 1.3 that $x_{n} \rightarrow \bar{x}$.

Next, we consider another case.

Case 2. Suppose that the sequence $\left\{\left\|x_{n}-\bar{x}\right\|\right\}$ is not eventually decreasing. There exists a subsequence $\left\{\left\|x_{n_{i}}-\bar{x}\right\|\right\}$ such that $\left\|x_{n_{i}}-\bar{x}\right\| \leq\left\|x_{n_{i}+1}-\bar{x}\right\|$ for all $i \geq 0$. We define an integer sequence $k(n)$ as in Lemma 1.5. By use of (2.8), we have

$$
\begin{aligned}
& \left\|x_{k(n)+1}-\bar{x}\right\|^{2} \\
& \leq \quad\left(1-\frac{2 \alpha_{k(n)}\left(1-\beta_{k(n)}\right)(1-\beta)}{1+\alpha_{k(n)}(1-\beta)}\right)\left\|x_{k(n)}-\bar{x}\right\|^{2} \\
& \quad+\frac{2 \alpha_{k(n)}\left(1-\beta_{k(n)}\right)}{1+\alpha_{k(n)}(1-\beta)}\left(f(\bar{x})-\bar{x}, y_{k(n)}-\bar{x}\right) \\
& \quad-\left(1-\beta_{k(n)}\right) r_{k(n)}\left(2 \alpha-r_{k(n)}\right)\left\|A y_{k(n)}-A \bar{x}\right\|^{2} \\
& \quad-\left(1-\beta_{k(n)}\right)\left\|\left(I-J_{\left.r_{k(n)}\right)}\right)\left(y_{k(n)}-r_{k(n)} A y_{k(n)}\right)-\left(I-J_{r_{k(n)}}\right)\left(\bar{x}-r_{k(n)} A \bar{x}\right)\right\|^{2} .
\end{aligned}
$$

It follows that

$$
\lim _{n \rightarrow \infty}\left\|y_{k(n)}-J_{r_{k(n)}}\left(y_{k(n)}-r_{k(n)} A y_{k(n)}\right)\right\|=0 .
$$

Hence, we have $\lim \sup _{n \rightarrow \infty}\left\langle f(\bar{x})-\bar{x}, y_{k(n)}-\bar{x}\right\rangle \leq 0$. In view of (2.9), we find that

$$
\lim _{n \rightarrow \infty}\left\|x_{k(n)}-\bar{x}\right\|=0
$$

Note that

$$
\begin{aligned}
\left\|y_{k(n)+1}-\bar{x}\right\| \leq & \left\|y_{k(n)+1}-x_{k(n)+1}\right\|+\left\|x_{k(n)+1}-x_{k(n)}\right\|+\left\|x_{k(n)}-\bar{x}\right\| \\
\leq & \alpha_{k(n)+1}\left\|f\left(x_{k(n)+1}\right)-x_{k(n)+1}\right\| \\
& +\left(1-\beta_{k(n)}\right)\left\|J_{r_{k(n)}}\left(y_{k(n)}-r_{k(n)} A y_{k(n)}\right)-x_{k(n)}\right\|+\left\|x_{k(n)}-\bar{x}\right\| \\
\leq & \alpha_{k(n)+1}\left\|f\left(x_{k(n)+1}\right)-x_{k(n)+1}\right\|+\left\|J_{r_{k(n)}}\left(y_{k(n)}-r_{k(n)} A y_{k(n)}\right)-y_{k(n)}\right\| \\
& +\alpha_{k(n)}\left\|f\left(x_{k(n)}\right)-x_{k(n)}\right\|+\left\|x_{k(n)}-\bar{x}\right\| .
\end{aligned}
$$

By use of (2.10), we find that $\lim _{n \rightarrow \infty}\left\|y_{n}-\bar{x}\right\|=0$. Since $\left\|x_{n}-\bar{x}\right\| \leq \alpha_{n}\left\|f\left(x_{n}\right)-x_{n}\right\|+\left\|y_{n}-\bar{x}\right\|$, we find that $\lim _{n \rightarrow \infty}\left\|x_{n}-\bar{x}\right\|=0$. This completes the proof.

Remark 2.5 Comparing Theorem 2.4 with the recent results announced in $[7,11]$ and [24], we have the following:

(i) Our proofs are different from theirs.

(ii) We remove the additional restriction $\sum_{n=1}^{\infty}\left|r_{n+1}-r_{n}\right|<\infty$.

\section{Applications}

In this section, we investigate solutions of equilibrium problems, variational inequalities and convex minimization problems, respectively. 
Let $F$ be a bifunction of $C \times C$ into $\mathbb{R}$, where $\mathbb{R}$ denotes the set of real numbers. Recall the following equilibrium problem:

Find $x \in C$ such that $F(x, y) \geq 0, \quad \forall y \in C$.

In this paper, we use $E P(F)$ to denote the solution set of the equilibrium problem.

To study equilibrium problems (3.1), we may assume that $F$ satisfies the following conditions:

(A1) $F(x, x)=0$ for all $x \in C$;

(A2) $F$ is monotone, i.e., $F(x, y)+F(y, x) \leq 0$ for all $x, y \in C$;

(A3) for each $x, y, z \in C$,

$$
\limsup _{t \downarrow 0} F(t z+(1-t) x, y) \leq F(x, y)
$$

(A4) for each $x \in C, y \mapsto F(x, y)$ is convex and weakly lower semi-continuous.

Lemma 3.1 [24] Let $C$ be a nonempty closed convex subset of a real Hilbert space H. Let $F$ be a bifunction from $C \times C$ to $\mathbb{R}$ which satisfies (A1)-(A4) and let $B$ be a multivalued mapping of $H$ into itself defined by

$$
B x= \begin{cases}\{z \in H: F(x, y) \geq\langle y-x, z\rangle, \forall y \in C\}, & x \in C, \\ \varnothing, & x \notin C .\end{cases}
$$

Then $B$ is a maximal monotone operator with the domain $D\left(B_{F}\right) \subset C, E P(F)=B^{-1}(0)$, and $T_{r} x=(I+r B)^{-1} x, \forall x \in H, r>0$, where $T_{r}$ is defined as

$$
T_{r} x=\left\{z \in C: F(z, y)+\frac{1}{r}\langle y-z, z-x\rangle \geq 0, \forall y \in C\right\} .
$$

Theorem 3.2 Let $C$ be a nonempty closed convex subset of $H$. Let $S: C \rightarrow C$ be a nonexpansive mapping with fixed points and let $f: C \rightarrow C$ be a $\beta$-contractive mapping. Let $A: C \rightarrow H$ be an $\alpha$-inverse-strongly monotone mapping and let $F$ be a bifunction from $C \times C$ to $\mathbb{R}$ which satisfies (A1)-(A4). Assume that $F(S) \cap E P(F)$ is not empty. Let $\left\{\alpha_{n}\right\}$ and $\left\{\beta_{n}\right\}$ be real number sequences in $(0,1)$ and let $\left\{r_{n}\right\}$ be a positive real number sequence in $(0,2 \alpha)$. Let $\left\{x_{n}\right\}$ be a sequence generated in the following process: $x_{1} \in C$ and

$$
\left\{\begin{array}{l}
y_{n}=\alpha_{n} f\left(x_{n}\right)+\left(1-\alpha_{n}\right) x_{n}, \\
x_{n+1}=\beta_{n} x_{n}+\left(1-\beta_{n}\right) S\left(I+r_{n} B\right)^{-1}\left(y_{n}-r_{n} A y_{n}\right), \quad \forall n \geq 1,
\end{array}\right.
$$

where $B$ is a mapping defined as in (3.2). Assume that the control sequences satisfy the following restrictions:

(a) $\lim _{n \rightarrow \infty} \alpha_{n}=0, \sum_{n=0}^{\infty} \alpha_{n}=\infty$;

(b) $0<\liminf _{n \rightarrow \infty} \beta_{n} \leq \limsup _{n \rightarrow \infty} \beta_{n}<1$;

(c) $0<a \leq r_{n} \leq b<2 \alpha$ and $\sum_{n=1}^{\infty}\left|r_{n}-r_{n-1}\right|<\infty$, 
where $a$ and $b$ are two real numbers. Then $\left\{x_{n}\right\}$ converges strongly to a point $\bar{x} \in F(S) \cap$ $E P(F)$, which is a unique solution to the following variational inequality:

$$
\langle f(\bar{x})-\bar{x}, p-\bar{x}\rangle \leq 0, \quad \forall p \in F(S) \cap E P(F) .
$$

Theorem 3.3 Let $C$ be a nonempty closed convex subset of $H$. Let $f: C \rightarrow C$ be a $\beta$ contractive mapping. Let $A: C \rightarrow H$ be an $\alpha$-inverse-strongly monotone mapping and Let $F$ be a bifunction from $C \times C$ to $\mathbb{R}$ which satisfies (A1)-(A4). Assume that $E P(F)$ is not empty. Let $\left\{\alpha_{n}\right\}$ and $\left\{\beta_{n}\right\}$ be real number sequences in $(0,1)$ and let $\left\{r_{n}\right\}$ be a positive real number sequence in $(0,2 \alpha)$. Let $\left\{x_{n}\right\}$ be a sequence generated in the following process: $x_{1} \in C$ and

$$
\left\{\begin{array}{l}
y_{n}=\alpha_{n} f\left(x_{n}\right)+\left(1-\alpha_{n}\right) x_{n}, \\
x_{n+1}=\beta_{n} x_{n}+\left(1-\beta_{n}\right)\left(I+r_{n} B\right)^{-1}\left(y_{n}-r_{n} A y_{n}\right), \quad \forall n \geq 1 .
\end{array}\right.
$$

Assume that the control sequences satisfy the following restrictions:

(a) $\lim _{n \rightarrow \infty} \alpha_{n}=0, \sum_{n=0}^{\infty} \alpha_{n}=\infty$;

(b) $0 \leq \beta_{n} \leq \bar{\beta}<1$;

(c) $0<a \leq r_{n} \leq b<2 \alpha$,

where $\bar{\beta}, a$, and $b$ are real numbers. Then $\left\{x_{n}\right\}$ converges strongly to a point $\bar{x} \in(A+B)^{-1}(0)$, which is also a unique solution to the following variational inequality:

$$
\langle f(\bar{x})-\bar{x}, p-\bar{x}\rangle \leq 0, \quad \forall p \in(A+B)^{-1}(0) .
$$

Let $g: H \rightarrow(-\infty,+\infty]$ be a proper convex lower semi-continuous function. Then the subdifferential $\partial g$ of $g$ is defined as follows:

$$
\partial g(x)=\{y \in H: g(z) \geq g(x)+\langle z-x, y\rangle, z \in H\}, \quad \forall x \in H .
$$

From Rockafellar [4], we know that $\partial g$ is maximal monotone. It is easy to verify that $0 \in$ $\partial g(x)$ if and only if $g(x)=\min _{y \in H} g(y)$. Let $I_{C}$ be the indicator function of $C$, i.e.,

$$
I_{C}(x)= \begin{cases}0, & x \in C, \\ +\infty, & x \notin C .\end{cases}
$$

Since $I_{C}$ is a proper lower semi-continuous convex function on $H$, we see that the subdifferential $\partial I_{C}$ of $I_{C}$ is a maximal monotone operator.

Lemma 3.4 [24] Let $C$ be a nonempty closed convex subset of $H$ and let $\operatorname{Proj}_{C}$ be the metric projection from $H$ onto $C$. Let $\partial I_{C}$ be the subdifferential of $I_{C}$, where $I_{C}$ is as defined in (3.3). Then $y=\left(I+\lambda \partial I_{C}\right)^{-1} x \Longleftrightarrow y=\operatorname{Proj}_{C} x, \forall x \in H, y \in C$,

Theorem 3.5 Let $C$ be a nonempty closed convex subset of $H$. Let $S: C \rightarrow C$ be a nonexpansive mapping with fixed points and let $f: C \rightarrow C$ be a $\beta$-contractive mapping. Let $A: C \rightarrow H$ be an $\alpha$-inverse-strongly monotone mapping. Assume that $F(S) \cap V I(C, A)$ is not empty. Let $\left\{\alpha_{n}\right\}$ and $\left\{\beta_{n}\right\}$ be real number sequences in $(0,1)$ and let $\left\{r_{n}\right\}$ be a positive 
real number sequence in $(0,2 \alpha)$. Let $\left\{x_{n}\right\}$ be a sequence generated in the following process: $x_{1} \in C$ and

$$
\left\{\begin{array}{l}
y_{n}=\alpha_{n} f\left(x_{n}\right)+\left(1-\alpha_{n}\right) x_{n}, \\
x_{n+1}=\beta_{n} x_{n}+\left(1-\beta_{n}\right) S \operatorname{Proj}_{C}\left(y_{n}-r_{n} A y_{n}\right), \quad \forall n \geq 1 .
\end{array}\right.
$$

Assume that the control sequences satisfy the following restrictions:

(1) $\lim _{n \rightarrow \infty} \alpha_{n}=0, \sum_{n=0}^{\infty} \alpha_{n}=\infty$;

(2) $0<\liminf _{n \rightarrow \infty} \beta_{n} \leq \limsup _{n \rightarrow \infty} \beta_{n}<1$;

(3) $0<a \leq r_{n} \leq b<2 \alpha$ and $\sum_{n=1}^{\infty}\left|r_{n}-r_{n-1}\right|<\infty$,

where $a$ and $b$ are two real numbers. Then $\left\{x_{n}\right\}$ converges strongly to a point $\bar{x} \in F(S) \cap$ $V I(C, A)$, which is a unique solution to the following variational inequality:

$$
\langle f(\bar{x})-\bar{x}, p-\bar{x}\rangle \leq 0, \quad \forall p \in F(S) \cap V I(C, A) .
$$

Proof Putting $B x=\partial I_{C}$, we find from Theorem 2.1 and Lemma 3.4 the desired conclusion immediately.

Theorem 3.6 Let $C$ be a nonempty closed convex subset of $H$. Let $f: C \rightarrow C$ be a $\beta$ contractive mapping and let $A: C \rightarrow H$ be an $\alpha$-inverse-strongly monotone mapping. Assume that $F \operatorname{VI}(C, A)$ is not empty. Let $\left\{\alpha_{n}\right\}$ and $\left\{\beta_{n}\right\}$ be real number sequences in $(0,1)$ and let $\left\{r_{n}\right\}$ be a positive real number sequence in $(0,2 \alpha)$. Let $\left\{x_{n}\right\}$ be a sequence generated in the following process: $x_{1} \in C$ and

$$
\left\{\begin{array}{l}
y_{n}=\alpha_{n} f\left(x_{n}\right)+\left(1-\alpha_{n}\right) x_{n} \\
x_{n+1}=\beta_{n} x_{n}+\left(1-\beta_{n}\right) \operatorname{Proj}_{C}\left(y_{n}-r_{n} A y_{n}\right), \quad \forall n \geq 1 .
\end{array}\right.
$$

Assume that the control sequences satisfy the following restrictions:

(a) $\lim _{n \rightarrow \infty} \alpha_{n}=0, \sum_{n=0}^{\infty} \alpha_{n}=\infty$;

(b) $0 \leq \beta_{n} \leq \bar{\beta}<1$;

(c) $0<a \leq r_{n} \leq b<2 \alpha$,

where $\bar{\beta}, a$, and $b$ are real numbers. Then $\left\{x_{n}\right\}$ converges strongly to a point $\bar{x} \in(A+B)^{-1}(0)$, which is also a unique solution to the following variational inequality:

$$
\langle f(\bar{x})-\bar{x}, p-\bar{x}\rangle \leq 0, \quad \forall p \in(A+B)^{-1}(0)
$$

Proof Putting $B=\partial I_{C}$, we find from Theorem 2.4 and Lemma 3.4 the desired conclusion immediately.

Let $W: H \rightarrow \mathbb{R}$ be a convex and differentiable function and $M: H \rightarrow \mathbb{R}$ is a convex function. Consider the convex minimization problem $\min _{x \in H}(W(x)+M(x))$. From [25], we know if $\nabla W$ is $\frac{1}{L}$-Lipschitz continuous, then it is $L$-inverse-strongly monotone. Hence, we have the following results.

Theorem 3.7 Let $W: H \rightarrow \mathbb{R}$ be a convex and differentiable function such that $\nabla W$ is $\frac{1}{L}$ Lipschitz continuous and let $M: H \rightarrow \mathbb{R}$ be a convex and lower semi-continuous function 
such that $(\nabla W+\partial M)^{-1}(0)$ is not empty. Let $f$ be a $\beta$-contractive mapping on $H$. Let $\left\{\alpha_{n}\right\}$ and $\left\{\beta_{n}\right\}$ be real number sequences in $(0,1)$ and let $\left\{r_{n}\right\}$ be a positive real number sequence in $(0,2 \alpha)$. Let $\left\{x_{n}\right\}$ be a sequence generated in the following process: $x_{1} \in C$ and

$$
\left\{\begin{array}{l}
y_{n}=\alpha_{n} f\left(x_{n}\right)+\left(1-\alpha_{n}\right) x_{n}, \\
x_{n+1}=\beta_{n} x_{n}+\left(1-\beta_{n}\right)\left(I+r_{n} M\right)^{-1}\left(y_{n}-r_{n} \nabla W y_{n}\right), \quad \forall n \geq 1 .
\end{array}\right.
$$

Assume that the control sequences satisfy the following restrictions:

(a) $\lim _{n \rightarrow \infty} \alpha_{n}=0, \sum_{n=0}^{\infty} \alpha_{n}=\infty$;

(b) $0 \leq \beta_{n} \leq \bar{\beta}<1$;

(c) $0<a \leq r_{n} \leq b<2 \alpha$,

where $\bar{\beta}, a$, and $b$ are real numbers. Then $\left\{x_{n}\right\}$ converges strongly to a point $\bar{x} \in(\nabla W+$ $\partial M)^{-1}$, which is also a unique solution to the following variational inequality:

$$
\langle f(\bar{x})-\bar{x}, p-\bar{x}\rangle \leq 0, \quad \forall p \in(\nabla W+\partial M)^{-1} .
$$

Proof Putting $A=\nabla W$ and $B=\partial M$, we find from Theorem 2.4 the desired conclusion immediately.

\section{Conclusions}

In this paper, we study a convex feasibility problem via two monotone mappings and a nonexpansive mapping. The common solution is also a unique solution of another variational inequality. The restrictions imposed on the sequence $\left\{r_{n}\right\}$ are mild. The results presented in this paper mainly improve the corresponding results in [7] and [11].

\section{Competing interests}

The author declares that they have no competing interests.

\section{Acknowledgements}

The author is very grateful to the editor and anonymous reviewers' suggestions which improved the contents of the article.

Received: 25 August 2014 Accepted: 5 December 2014 Published: 06 Jan 2015

\section{References}

1. Martinet, B: Regularisation d'inéquations variationelles par approximations successives. Rev. Fr. Inform. Rech. Oper. 4, 154-158 (1970)

2. Martinet, B: Determination approchée d'un point fixe d'une application pseudo-contractante. C. R. Acad. Sci. Paris Sér. A-B 274, 163-165 (1972)

3. Rockafellar, RT: Monotone operators and the proximal point algorithm. SIAM J. Control Optim. 14, 877-898 (1976)

4. Rockafellar, RT: Augmented Lagrangians and applications of the proximal point algorithm in convex programming. Math. Oper. Res. 1, 97-116 (1976)

5. Lions, PL, Mercier, B: Splitting algorithms for the sum of two nonlinear operators. SIAM J. Numer. Anal. 16, 964-979 (1979)

6. Passty, GB: Ergodic convergence to a zero of the sum of monotone operators in Hilbert space. J. Math. Anal. Appl. 72, 383-390 (1979)

7. Cho, SY, Qin, X, Wang, L: Strong convergence of a splitting algorithm for treating monotone operators. Fixed Point Theory Appl. 2014, Article ID 94 (2014)

8. Zegeye, $\mathrm{H}$, Shahzad, N: Strong convergence theorem for a common point of solution of variational inequality and fixed point problem. Adv. Fixed Point Theory 2, 374-397 (2012)

9. Cho, SY, Kang, SM: On iterative solutions of a common element problem. J. Nonlinear Funct. Anal. 2014, Article ID 3 (2014)

10. Zhang, M: An algorithm for treating asymptotically strict pseudocontractions and monotone operators. Fixed Point Theory Appl. 2014, Article ID 52 (2014)

11. Qin, $X, C h o, S Y$, Wang, L: A regularization method for treating zero points of the sum of two monotone operators Fixed Point Theory Appl. 2014, Article ID 75 (2014) 
12. Hecai, Y: On weak convergence of an iterative algorithm for common solutions of inclusion problems and fixed point problems in Hilbert spaces. Fixed Point Theory Appl. 2013, Article ID 155 (2013)

13. Zhao, J, Zhang, Y, Yang, Q: Modified projection methods for the split feasibility problem and the multiple-sets split feasibility problem. Appl. Math. Comput. 219, 1644-1653 (2012)

14. Qin, X, Cho, SY, Wang, L: Convergence of splitting algorithms for the sum of two accretive operators with applications. Fixed Point Theory Appl. 2014, Article ID 166 (2014)

15. Yu, L, Liang, M: Convergence of iterative sequences for fixed point and variational inclusion problems. Fixed Point Theory Appl. 2011, Article ID 368137 (2011)

16. Yang, S: Zero theorems of accretive operators in reflexive Banach spaces. J. Nonlinear Funct. Anal. 2013, Article ID 2 (2013)

17. Qin, X, Su, Y: Approximation of a zero point of accretive operator in Banach spaces. J. Math. Anal. Appl. 329, 415-424 (2007)

18. Barbu, V: Nonlinear Semigroups and Differential Equations in Banach Space. Noordhoff, Groningen (1976)

19. Suzuki, T: Strong convergence of Krasnoselskii and Mann's type sequences for one-parameter nonexpansive semigroups without Bochner integrals. J. Math. Anal. Appl. 305, 227-239 (2005)

20. Liu, LS: Ishikawa and Mann iterative process with errors for nonlinear strongly accretive mappings in Banach spaces. J. Math. Anal. Appl. 194, 114-125 (1995)

21. Lopez, G, Marquez, VM, Wang, F, Xu, HK: Forward-backward splitting methods for accretive operators in Banach spaces. Abstr. Appl. Anal. 2012, Article ID 109236 (2012)

22. Mainge, PE: Strong convergence of projected subgradient methods for nonsmooth and nonstrictly convex minimization. Set-Valued Anal. 16, 899-912 (2008)

23. Browder, FE: Nonexpansive nonlinear operators in a Banach space. Proc. Natl. Acad. Sci. USA 54, 1041-1044 (1965)

24. Takahashi, S, Takahashi, W, Toyoda, M: Strong convergence theorems for maximal monotone operators with nonlinear mappings in Hilbert spaces. J. Optim. Theory Appl. 147, 27-41 (2010)

25. Baillon, JB, Haddad, G: Quelques propriétés des opérateurs angle-bornés et cycliquement monotones. Isr. J. Math. 26 137-150 (1977)

10.1186/1029-242X-2015-2

Cite this article as: Zhang: Some results on a viscosity splitting algorithm in Hilbert spaces. Journal of Inequalities and Applications 2015, 2015:2

\section{Submit your manuscript to a SpringerOpen ${ }^{\circ}$ journal and benefit from:}

- Convenient online submission

- Rigorous peer review

- Immediate publication on acceptance

- Open access: articles freely available online

- High visibility within the field

- Retaining the copyright to your article 